\title{
Der (un)eingeschlagene Pfad
}

\author{
Edy Portmann ${ }^{1}$
}

Angenommen: 12. Oktober 2021 / Online publiziert: 28. Oktober 2021

(c) Der/die Autor(en) 2021

\section{Zusammenfassung}

In einer ersten, durch den Physiker Freeman Dyson inspirierten „Common-Sense“ Kolumne vergleicht der Wirtschaftsinformatiker Edy Portmann die Geschichte von Analog- mit der von Digitalcomputer und fordert abschließend, mit einem gewagten Blick in die Zukunft, sein Schicksal als humanistischer Prophet heraus.

Im Jungpaläolithikum vor etwa 20.000 Jahren wurde im Norden des Eduardsees in der heutigen Demokratischen Republik Kongo der wohl erste „Computer“ hergestellt, den wir uns als Art prähistorischer Taschenrechner vorstellen können. Heute kann man den „Ishango“-Knochen, der 1960 entdeckt wurde, im Museum für Naturwissenschaften in Brüssel besichtigen. Er weist Markierungen auf, die entweder Strichmännchen, Primzahlen oder einen Mondkalender darstellen könnten. Hier möchte ich diesen analogen Computer, der auf kontinuierlichen Zahlenrepräsentationen basiert, mit Digitalcomputer, die auf diskreten Repräsentationen basieren, vergleichen, sowie einen Blick in die $\mathrm{Zu}-$ kunft wagen.

Noch in den 1930er-Jahren standen uns 5 Arten von Rechnern zur Verfügung, 2 waren digital und 3 analog. Im Jahr 1936, schrieb dann der Mathematiker Alan Turing, als quasi erster Informatiker, einen Artikel über berechenbare Zahlen, der die Leistungsfähigkeit des digitalen Rechnens als logische Konstruktion aufzeigte. Nach seiner Publikation kamen Analogcomputer langsam aus der Mode, da diese komplex(er) waren (als digitale). Heute sind wir mittlerweile so stark in der Welt der Digitalcomputer angekommen, sodass es uns schwerfällt, zu sehen, dass es auch analog gehen könnte. Aber eventuell ist analog nicht nur unsere Vergangenheit, sondern auch unsere Zukunft?

Edy Portmann

edy.portmann@unifr.ch
Bereits 1981 bewiesen die Mathematiker Marian PourEl und Ian Richards von der Universität Minnesota nämlich ein Theorem, das mathematisch präzise aussagt, dass analoge Computer leistungsfähiger sind als digitale. Sie geben in einem Artikel ein Beispiel für Zahlen, die sich mit Digitalcomputern nicht berechnen lassen, aber mit einer sehr einfachen Art von Analogcomputern berechenbar sind. Turing hatte in seinem Artikel berechenbare Zahlen als solche definiert, die mit digitalen Rechnern berechnet werden können. Die von Pour-El und Richards diskutierten Zahlen sind dagegen analog und somit nicht nach Turing berechenbar. Aber was bedeutet das?

Ein analoger Computer arbeitet mit kontinuierlichen, während ein digitaler Computer mit diskreten, also abzählbaren Zahlen arbeitet, bei denen, im Gegensatz zu kontinuierlichen, Stetigkeit keine Rolle spielt. Der Computer von Pour-El und Richards charakterisiert eine Feldausbreitung durch das Raumzeitkontinuum, das einer linearen Wellengleichung folgt. In ihrem Artikel zeigen die beiden, dass das Feld auf einen Punkt fokussiert werden kann, sodass die Stärke des Feldes an diesem Punkt nicht von einem digitalen Computer berechnet, aber von einem einfachen analogen Gerät gemessen werden kann. Mit ihrem Artikel haben sie für Analogcomputer das gleiche geleistet wie Turing 45 Jahre zuvor für Digitalcomputer.

In einem Auftritt am University College Dublin, der als Basis diese Kolumne dient, fragte der Physiker Freeman Dyson, ein Mathematikgenie, das zum Technikvisionär avancierte, ob Gehirne analog oder digital seien. Er legte dar, dass Kunstschaffende wie Stanley Kubrick mit dem Film 2001: Odyssee im Weltraum, in dem Bewusstsein von einem Gehirn in einen Computer heruntergeladen wird, ein

1 Universität Freiburg, Bd de Pérolles 90, 1700 Freiburg, Schweiz 
Zukunftsbild vom digitalen und Fred Hoyle mit dem Buch Black Cloud, in dem Bewusstsein in eine schwarze Wolke, die Erinnerungen in Magnetfeldern kodiert, hochgeladen wird, vom Analogen vermittelt haben.

Gemäß Dyson kann ein Herunterladen in einen digitalen Computer zu einem Verlust unserer analogen, menschlichen Qualitäten führen. „Ich habe keine Lust, das Experiment selbst zu versuchen“, scherzt Dyson in seinem Auftritt, aber, ,wenn es für uns in Zukunft an der Zeit ist, uns an das Universum anzupassen und unsere Gewohnheiten aufzugeben, sollten wir uns in schwarze Wolken hochanstatt uns in Digitalchips herunterladen." Wenn ich mich entscheiden müsste, würde ich ihm folgen. Und Sie?

Funding Open access funding provided by University of Fribourg

Open Access Dieser Artikel wird unter der Creative Commons Namensnennung 4.0 International Lizenz veröffentlicht, welche die Nutzung, Vervielfältigung, Bearbeitung, Verbreitung und Wiedergabe in jeglichem Medium und Format erlaubt, sofern Sie den/die ursprünglichen Autor(en) und die Quelle ordnungsgemäß nennen, einen Link zur Creative Commons Lizenz beifügen und angeben, ob Änderungen vorgenommen wurden.

Die in diesem Artikel enthaltenen Bilder und sonstiges Drittmaterial unterliegen ebenfalls der genannten Creative Commons Lizenz, sofern sich aus der Abbildungslegende nichts anderes ergibt. Sofern das betreffende Material nicht unter der genannten Creative Commons Lizenz steht und die betreffende Handlung nicht nach gesetzlichen Vorschriften erlaubt ist, ist für die oben aufgeführten Weiterverwendungen des Materials die Einwilligung des jeweiligen Rechteinhabers einzuholen.

Weitere Details zur Lizenz entnehmen Sie bitte der Lizenzinformation auf http://creativecommons.org/licenses/by/4.0/deed.de.

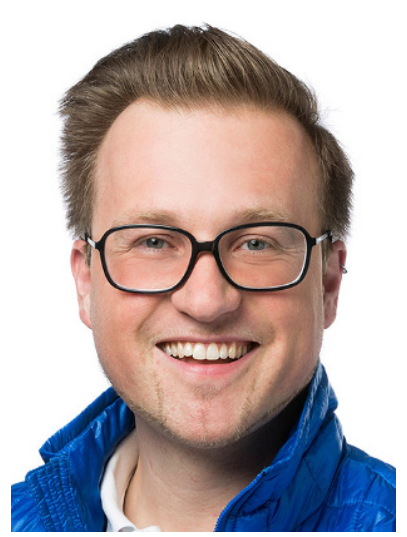

